\title{
Reset control of boost converters
}

\author{
Unnikrishnan Raveendran Nair ${ }^{1}$, Ramon Costa-Castelló ${ }^{1}$ and Alfonso Baños ${ }^{2}$
}

\begin{abstract}
The goal of this work is to apply the framework of reset systems, in particular PI+CI controllers, in the controller design for power converters. While the PI+CI controller has been applied in several industrial applications, the application of such controllers in fast electrical systems especially power electronic converters appears to be new. The main motivations for this proposal are performance superiority of these controllers and the ability to produce a fast flat response without any overshoot for a step input. Another factor that influenced the use of such controller is the relatively simple design equations, which enables plug and play capability. The flat responses are highly interesting from the perspective of power converters especially when they are connected to power grids.
\end{abstract}

\section{INTRODUCTION}

Climate changes from global warming by increased usage of fossil fuels have resulted in stricter sanctions being imposed by many governmental organisations to reduce greenhouse emissions. A major outcome of this has been the influx of renewable sources like wind and solar in power grids. The issue with these sources are the pulsating nature of its power and the type of power generated (DC instead of $\mathrm{AC}$ ). The pulsating power issue is overcome using electrical storages like fuel cell-electrolyser system, batteries and super capacitors (SC)[1]. The major issue, though will be the DC nature of the power generated by these and voltage levels at the output of these devices. Switch mode power converters therefore, play a major role in the grid connection of these renewable-storage hybrid systems providing power conversion and conditioning capability [2][3].

A robust, optimal controller design for these power converters plays a major role in the efficient and reliable integration of the renewable sources in modern grids. Non linear control using sliding mode techniques have been proposed for converters[4][5][6]. Despite this, most of the converters have relied heavily on the classical PI controllers due to its simple design techniques and robust nature. These converters should have fast response to varying load demands without introducing significant voltage flicker in the grids. The PI controllers can result in overshoot at converter output for fast responses resulting in voltage flickers in the grid. This overshoot is avoided if the speed of response is reduced.

\footnotetext{
*This work was partially supported, including FEDER co-funding, by the Spanish Ministerio de Educacin project DPI2015-69286-C3-2-R (MINECO/FEDER), project DPI2013-47100-C2-1-P, and project DPI201679278-C2-1-R

${ }^{1}$ Unnikrishnan Raveendran Nair, Ramon Costa-Castelló are with Institut de Robòtica i Informòtica industrial, Universitat Politècnica de Catalunya, C/Pau Gargallo 5, 08028 Barcelona, Spain uraveendran@iri.upc.edu, ramon. costa@upc.edu

${ }^{2}$ Alfonso Baños with the Departmento. Informática y Sistemas, Universidad de Murcia, Spain abanos@um.es
}

Therefore, there is always a trade-off between the speed of response and the quality of power output. The PI is usually tuned to meet grid codes which lays guidelines for speed of response and maximum overshoot permissible. Nevertheless a flat, fast response to the varying load demands is ideal for improved power quality. Higher order sliding mode controllers are capable of achieving such responses but result in more complex controllers[4]. Reset controllers which employ partial resetting of its states is an option to achieve fast, flat response. Reset controllers are interesting since they are modifications of classical linear controllers and provide simple realisations[7].

Reset controllers are hybrid systems. The earliest instance has been the Clegg Integrator (CI) introduced by J C Clegg. This CI was used in servo systems and used an integrator that resets to zero at zero error to reduce overshoot [8]. This was followed by the First order reset element (FORE) with the design procedure of the same outlined in [9][10]. The $\mathrm{PI}+\mathrm{CI}$ is another reset controller using the linear PI controller along with the CI. The CI as a standalone controller cannot have zero steady state error unless the plant is an integrator. The PI+CI uses the PI to ensure zero steady-state error while the CI improves controller performance by enabling fast response without overshoot. These performance levels are beyond the capabilities of PI controller [7],[11],[12] and makes it ideal for power converters.

Most of the works in PI+CI controllers have focused on defining reset instants through reset laws and reset ratios (discussed in upcoming sections) [7][11] to improve various performance parameters [13]. Model predictive control strategy has also been used to define reset law for uncertain system as presented in [14]. Studies have also been done to analyse the stability and robustness of the reset systems. Describing functions have been used to analyse the stability and robustness but they have proved to fail in certain cases [12]. More conclusive techniques employing linear matrix inequalities, Lyapunov stability criteria [12] and frequency domain methods have also been developed [15].

PI+CI controller has been employed in applications like $\mathrm{pH}$ in-line control [11], industrial wafer scanners [16], control of industrial heat exchangers[17] which are inherently slow systems. The application of such controllers in fast electrical systems especially power converters has not been proposed according to authors knowledge. As such this work tries to apply the PI+CI design method developed in [11] to a controller for a DC-DC boost converter. The main challenge and contribution will be in ensuring that the PI+CI controller, designed for obtaining a flat response in first order system [11], provides the same performance in higher order systems 


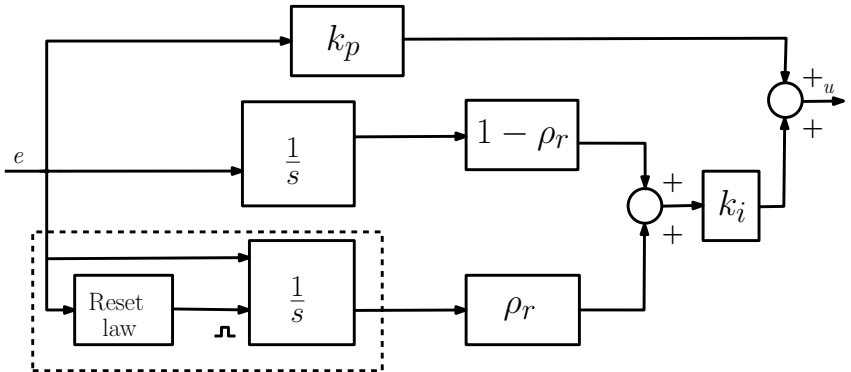

Fig. 1. PI+CI controller setup.

presented by the converter. As such this work can be viewed as an application of $\mathrm{PI}+\mathrm{CI}$ controller to power converters in order to ensure its feasibility for grid connected systems.

The rest of the paper is organised as follows. The section II lays out the preliminaries where the PI+CI system equations and modelling of DC-DC boost converter is presented. The section III presents design procedure of the controller. The proposed controller's simulation results are presented and discussed in section IV. Finally the conclusion and future work possibilities are outlined in section V.

\section{PReliminaries}

\section{A. PI+CI compensator}

The PI+CI controller is shown in Fig.1. The PI controller has the CI (dashed rectangle) in parallel. The block $\rho_{r}$ is the reset ratio and defines the percentage of integral action reset through CI. If $\rho_{r}$ is 0 it is a normal PI controller with no reset, which will be referred to as $P I_{\text {base }}$ henceforth, while a $\rho_{r}$ of 1 is a full reset $\mathrm{P}+\mathrm{CI}$ system. The design problem will be to find $\rho_{r}$ between $0-1$ which gives the best controller performance for reference tracking. The reset law defines the instances of reset of CI. Reset law can be fixed [7] or varying [11] based on a function of the system states. The simplest $\mathrm{PI}+\mathrm{CI}$ controller with fixed reset instance at zero error and constant reset ratio is defined in state-space form by [12][7]

$$
P I+C I=\left\{\begin{array}{lll}
\dot{\mathbf{x}}_{\mathbf{r}}(\mathbf{t})=\mathbf{A}_{\mathbf{r}} \mathbf{x}_{\mathbf{r}}(\mathbf{t})+\mathbf{B}_{\mathbf{r}} e(t), & e(t) \neq 0 \\
\mathbf{x}_{\mathbf{r}}\left(\mathbf{t}^{+}\right)=\mathbf{A}_{\rho} \mathbf{x}_{\mathbf{r}}(\mathbf{t}), & e(t)=0 \\
u(t)=\mathbf{C}_{\mathbf{r}} \mathbf{x}_{\mathbf{r}}(\mathbf{t})+\mathbf{D}_{\mathbf{r}} e(t) &
\end{array}\right.
$$

where $\mathbf{x}_{\mathbf{r}}=\left[\begin{array}{ll}x_{i} & x_{c i}\end{array}\right]^{T}$ are the states of the controller defined by the integrator $\left(x_{i}\right)$ and CI $\left(x_{c i}\right)$ states, $\mathbf{x}_{\mathbf{r}}\left(\mathbf{t}^{+}\right)=\mathbf{x}_{\mathbf{r}}(\mathbf{t}+\varepsilon)$ with $\varepsilon \longrightarrow 0^{+}$and $e(t)$ is the error of the system. The matrices $\mathbf{A}_{\mathbf{r}}, \mathbf{B}_{\mathbf{r}}, \mathbf{C}_{\mathbf{r}}, \mathbf{D}_{\mathbf{r}}$ and $\mathbf{A}_{\rho}$ are

$$
\begin{array}{r}
\mathbf{A}_{\mathbf{r}}=\left[\begin{array}{ll}
0 & 0 \\
0 & 0
\end{array}\right], \quad \mathbf{B}_{\mathbf{r}}=\left[\begin{array}{l}
1 \\
1
\end{array}\right], \quad \mathbf{C}_{\mathbf{r}}=\frac{k_{p}}{\tau_{i}}\left[\begin{array}{ll}
1-\rho_{r} & \rho_{r}
\end{array}\right] \\
\mathbf{D}_{\mathbf{r}}=k_{p}, \quad \mathbf{A}_{\rho}=\left[\begin{array}{ll}
1 & 0 \\
0 & 0
\end{array}\right]
\end{array}
$$

The vector $\mathbf{x}_{\mathbf{r}}\left(\mathbf{t}^{+}\right)$defines the states of PI+CI controller after the instance of reset. $\mathbf{A}_{\rho}$ resets the CI state $\left(x_{c i}\right)$ while the integrator state $\left(x_{i}\right)$ remains the same.

The PI+CI controller can also be realised using variable reset ratio where the reset ratio will be a function of system

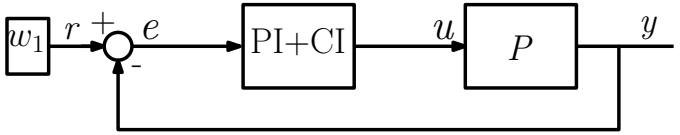

Fig. 2. PI+CI closed loop system.

state and error. These controllers provide improved performance over the constant reset ratio systems. The variable reset ratio systems is defined in state space form as [11]

$$
\begin{cases}\dot{\rho}_{\mathbf{r}}(\mathbf{t})=0, \dot{\mathbf{x}}_{\mathbf{r}}(\mathbf{t})=\mathbf{A}_{\mathbf{r}} \mathbf{x}_{\mathbf{r}}(\mathbf{t})+\mathbf{B}_{\mathbf{r}} e(t), & e(t) \neq 0 \\ \rho_{r}\left(t^{+}\right)=\mathcal{P}\left(\mathbf{x}_{\mathbf{r}}, e(t)\right), \mathbf{x}_{\mathbf{r}}\left(\mathbf{t}^{+}\right)=\mathbf{A}_{\rho} \mathbf{x}_{\mathbf{r}}(\mathbf{t}), & e(t)=0 \\ u(t)=\mathbf{C}_{\mathbf{r}}\left(\rho_{\mathbf{r}}(\mathbf{t})\right) \mathbf{x}_{\mathbf{r}}(\mathbf{t})+\mathbf{D}_{\mathbf{r}} e(t) & \end{cases}
$$

where $\mathcal{P}\left(\mathbf{x}_{\mathbf{r}}, e(t)\right)$ is the variable reset ratio function defined as $\mathcal{P}: \mathbb{R}^{2} \mathrm{x} \mathbb{R} \rightarrow \mathbb{R}$ and $C_{r}\left(\rho_{r}(t)\right)=\frac{k p}{\tau_{i}}\left[1-\rho_{r} \rho_{r}\right]$.

The performance evaluation of the fixed and variable reset ratio PI+CI discussed above is provided in [7] and [11] respectively. The variable reset ratio system is capable of achieving a flat response for reference tracking in a first order system irrespective of the design of $P I_{b a s e}$. The constant reset ratio system has improved performance over the base PI system but is not capable of flat response like the variable reset ratio systems. It is capable of reaching the steady-state at the instance of second zero crossing of the system output [7]. The advantage that the variable reset ratio system posses over the constant ratio system has prompted the use of such a system (3) in the control of power converters.

\section{B. Reset control system}

The Fig. 2 shows a single input single output plant controlled by the PI+CI controller. The plant $(\mathrm{P})$ under control is defined as

$$
\begin{aligned}
\dot{\mathbf{x}}_{\mathbf{p}}(\mathbf{t}) & =\mathbf{A}_{\mathbf{p}} \mathbf{x}_{\mathbf{p}}(\mathbf{t})+\mathbf{B}_{\mathbf{p}} u(t), \\
y(t) & =\mathbf{C}_{\mathbf{p}}(\mathbf{t}) \mathbf{x}_{\mathbf{p}}(\mathbf{t})
\end{aligned}
$$

where $\mathbf{x}_{\mathbf{p}} \in \mathbb{R}^{n_{p}}$. In this work, the main control goal will be the tracking of constant references, thus there is only an exogenous signal $w_{1}$ as shown in Fig. 2. It is assumed that $w_{1}$ is a Bohl function and thus, the input generator $w_{1}$ is defined by

$$
\begin{aligned}
\dot{w}_{1}(t) & =\mathbf{A}_{\mathbf{1}} w_{1}(t) \\
r(t) & =\mathbf{C}_{\mathbf{1}} w_{1}(t)
\end{aligned}
$$

where $w_{1} \in \mathbb{R}^{n 1}$.

The closed-loop system of Fig. 2 with variable reset ratio controller (3) and plant above (4)-(5) is then modelled using the framework expressed in [18] [11] in state space form as

$$
\begin{cases}\dot{\rho}_{r}(t)=0, \dot{\mathbf{x}}(\mathbf{t})=\mathbf{A}(\mathbf{t}) \mathbf{x}(\mathbf{t}), & \mathbf{x}(\mathbf{t}) \notin \mathcal{M} \\ \rho_{r}\left(t^{+}\right)=\mathcal{P}(x(t)), \mathbf{x}\left(\mathbf{t}^{+}\right)=\mathbf{A}_{\mathbf{R}} \mathbf{x}(\mathbf{t}), & \mathbf{x}(\mathbf{t}) \in \mathcal{M} \\ y(t)=\mathbf{C x}(\mathbf{t}) & \end{cases}
$$

where $\mathbf{x}$ defines states of the closed loop system defined by $\left[\mathbf{x}_{\mathbf{p}}, \mathbf{x}_{\mathbf{r}}, w_{1}\right]$. The total number of states will then be $n_{p}+$ $2+n_{1}$. The matrices $\mathbf{A}, \mathbf{C}, \mathbf{A}_{\mathbf{R}}$ are defined as

$$
\mathbf{A}(\mathbf{t})=\left[\begin{array}{ccc}
\mathbf{A}_{\mathbf{p}}-\mathbf{B}_{\mathbf{p}} \mathbf{D}_{\mathbf{r}} \mathbf{C}_{\mathbf{p}} & \mathbf{B}_{\mathbf{p}} \mathbf{C}_{\mathbf{r}}\left(\rho_{\mathbf{r}}(\mathbf{t})\right) & \mathbf{B}_{\mathbf{p}} \mathbf{D}_{\mathbf{r}} \mathbf{C}_{\mathbf{1}} \\
-\mathbf{B}_{\mathbf{r}} \mathbf{C}_{\mathbf{p}} & \mathbf{A}_{\mathbf{r}} & \mathbf{B}_{\mathbf{r}} \mathbf{C}_{\mathbf{1}} \\
0 & 0 & \mathbf{A}_{\mathbf{1}}
\end{array}\right]
$$




$$
\mathbf{C}=\left(\begin{array}{lll}
\mathbf{C}_{\mathbf{p}} & 0 & 0
\end{array}\right), \quad \mathbf{A}_{\mathbf{R}}=\operatorname{diag}\left(\mathbf{I}_{\mathbf{n}_{\mathbf{p}}}, \mathbf{A}_{\rho}, \mathbf{I}_{\mathbf{n}_{1}}\right)
$$

where $I$ is identity matrix of dimension defined by the subscript. The set $\mathcal{M}$ is defined as

$$
\mathcal{M}=\left\{\mathbf{x}(\mathbf{t}) \in \mathbb{R}^{n}: \mathbf{C}_{\mathcal{M}}(\mathbf{t}) \mathbf{x}(\mathbf{t})=0\right\}
$$

where $\mathbf{C}_{\mathcal{M}}(\mathbf{t})=\left(\begin{array}{llll}-\mathbf{C}_{\mathbf{p}} & 0 & \mathbf{C}_{\mathbf{1}} & 0\end{array}\right)$. The framework of modelling hybrid systems defined in [18] uses concept of flow and jump sets. The flow set is the set of values of system states where the system equations are defined by a first order differential equation referred to as flow map. The jump set on the contrary defines set of state values where a sudden jump (reset) in the state values occur and is defined by an impulse equation (in this specific case the reset equation). Based on this in (6) the set $\mathcal{M}$ is the jump set while the complementary of this set is the flow set. The system defined by (6) is also valid for the PI+CI systems with constant reset ratio. The only difference is that $\mathbf{A}$ matrix (7) will have a constant term $\mathbf{C}_{\mathbf{r}}$ instead of $\mathbf{C}_{\mathbf{r}}\left(\rho_{\mathbf{r}}(\mathbf{t})\right)$. Although it will not be included here, a rigorous well-posedness analysis of the closed-loop system (6), including existence, unicity of solutions, continuous dependence on initial condition and robustness to measurement noise, may be performed by a direct application of results in [11] and [19].

\section{Modelling of DC-DC boost converter}

The converter used here is the DC-DC boost converter which operates in single quadrant, shown in Fig.3. The converter boosts the input voltage $\left(v_{d c}\right)$ which can be from PV panels, batteries or SC to a higher voltage level $\left(v_{b u s}\right)$. The inductor $l_{1}$ and capacitor $c_{1}$ form input filter while $l_{2}$ is the inductor necessary for the boost converter operation [20]. The resistors $r_{1}$ and $r_{2}$ are the effective series resistance (ESR) values of $l_{1}$ and $l_{2}$ respectively. The detailed explanation on the working principle of the converter is not provided here as its beyond the scope of this paper. This can be found in detail in [20].

The converter is a switching system where the DC-DC conversion is achieved by the switching of an appropriate device, MOSFET or IGBT, by using gate signals. Therefore, a linearised model of the switched system is needed for the controller design. The linearising is done by using the average voltage across the switch in the converter equations given by $v_{c}=d^{\prime} v_{\text {bus }}$ where $d^{\prime}=1-d$ with $d$ the duty cycle of converter. The system equations applying Kirchoff voltage and current law is given by

$$
\begin{aligned}
& l_{1} \frac{i_{1}}{d t}=v_{d c}-v_{1}-r_{1} i_{1} \\
& l_{2} \frac{i_{2}}{d t}=v_{1}-v_{c}-r_{2} i_{2} \\
& c_{1} \frac{v_{1}}{d t}=i_{1}-i_{2} .
\end{aligned}
$$

In the above equations, there are two independent parameters $v_{d c}$ and $v_{c}$ forming the system input. Therefore, for a two input system defined above the the current equation $\left(i_{2}\right)$ is written as (10) (shown at the bottom of page).

In order to further simplify the above system a transformation is proposed. A new variable $v_{m 2}$ is defined given by

$$
V_{m 2}(s)=\frac{V_{d c}(s)}{l_{1} c_{1} s^{2}+r_{1} c_{1} s+1}-V_{c}(s) .
$$

The above transformation will allow the DC-DC converter of (9)-(10) to be modelled using a transfer function as shown in (12) below.

Based on (12), the controller will generate $v_{m 2}$ from which $v_{c}$ value can be extracted (10). This variable change also provides the advantage that there will always be a feed forward present even at the starting condition which will help limit large in-rush currents. The $v_{c}$ value will be applied to a modulator to generate the suitable gate signals.

\section{DESIgN OF VARIABLE RESET RATIO PI+CI FOR THE BOOST CONVERTER}

The design of the PI+CI controller for a third order system defined by (12) can be challenging especially considering the fact that the objective of the design problem is to obtain a flat response. The flat response achieved in [11] was for a first order system. This is possible since the error equation can be made to zero at the first zero crossing instant by using an appropriate value for $\rho_{r}$ [11] in first order systems. In higher order systems this is not the case. In [7] a method is outlined for the design of PI+CI controller with constant reset ratio for higher order systems using a first order equivalent of the system using half rule proposed in [21]. The application of this half rule for the model reduction in the boost converter can be tedious with the presence of complex conjugate pole and zero pairs in the system. Moreover, the reduction to first order will not guarantee a flat response. The design of $\mathrm{PI}+\mathrm{CI}$ for a second order system is outlined in [11] using the minimisation of $\mathrm{H}_{2}$-norm to obtain the optimal value for $\rho_{r}$. This resulted in the improved performance of the system compared to the $P I_{b a s e}$ system but the flat response in the

$$
I_{2}(s)=\frac{V_{d c}(s)-\left(c_{1} l_{1} s^{2}+c_{1} r_{1} s+1\right) V_{c}(s)}{l_{1} l_{2} c_{1} s^{3}+c_{1}\left(l_{1} r_{2}+l_{2} r_{1}\right) s^{2}+\left(c_{1} r_{1} r_{2}+l_{1}+l_{2}\right) s+\left(r_{1}+r_{2}\right)}
$$

$$
C(s)=\frac{I_{2}(s)}{V_{m 2}(s)}=\frac{c_{1} l_{1} s^{2}+c_{1} r_{1} s+1}{l_{1} l_{2} c_{1} s^{3}+c_{1}\left(l_{1} r_{2}+l_{2} r_{1}\right) s^{2}+\left(c_{1} r_{1} r_{2}+l_{1}+l_{2}\right) s+\left(r_{1}+r_{2}\right)}
$$




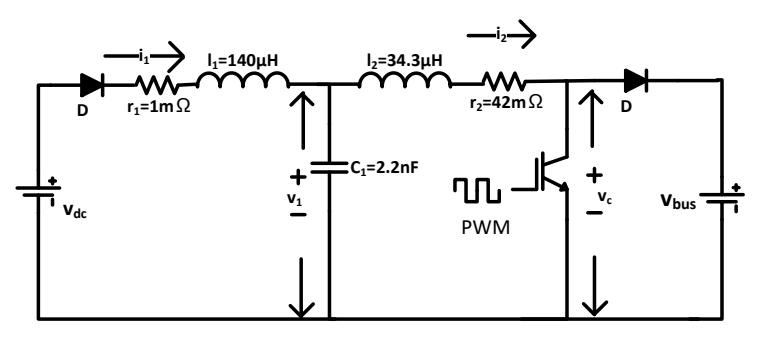

Fig. 3. Boost converter topology.

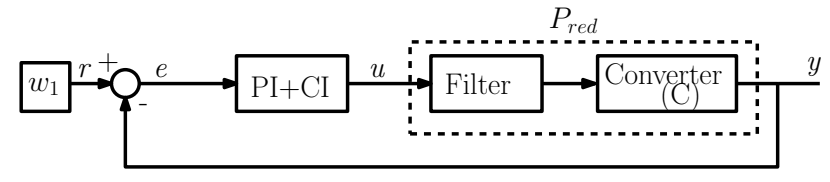

Fig. 4. Block diagram representation of the system with filter for compensation.

output was not obtained. It should therefore be concluded, that the presence of higher order dynamics in the system will result in a deviation from the flat response expected.

In order to ensure a flat response a two step process is proposed here. In the first step, a suitable compensation technique is defined to cancel the complex pole zero pairs. This will result in a real pole for the system. In the second step, a PI+CI controller with variable reset ratio can be designed by considering the system as an equivalent first order system to obtain a flat response in the output.

\section{A. Pole zero compensation}

The boost converter modelled in the previous section, (12), has the poles at $-498 \pm 4030 i,-254$ and zeros at $-35.6 \pm 1800 i$ for the component values used in the converter shown in Fig.3. These poles and zeros are compensated by adding a filter before the plant. The filter should be designed with poles at $-35.60 \pm 1800 i$ and zeros at $-498 \pm 4030 i$. This ensures cancellation of the converter complex conjugate pole-zero pairs resulting in an equivalent first order system (filter+converter) seen by the controller. The transfer function of the designed filter is

$$
F_{\text {filter }}(s)=\frac{s^{2}+991.4 s+16240000}{5.013 s^{2}+357.3 s+16240000} .
$$

The Fig. 5 shows Bode plots of the boost converter and filter designed. The filter compensates the phase shift and resonant peaks generated by the complex conjugate pole zero pairs of the converter and can be observed in Fig. 5 .

The Bode plot of the converter-filter system in open loop is shown in Fig.6. In comparison with the converter Bode plot presented in Fig. 5 it can be seen that the behaviour of the combined system is very similar to that of a first order plant. The converter-filter system can now be effectively modelled as a first order plant. The DC gain is calculated and remaining uncompensated pole at $-254 \mathrm{rad} / \mathrm{s}$ of the original system is used to define the reduced first order model of the system:

$$
P_{\text {red }}(s)=\frac{5826}{s+254}
$$

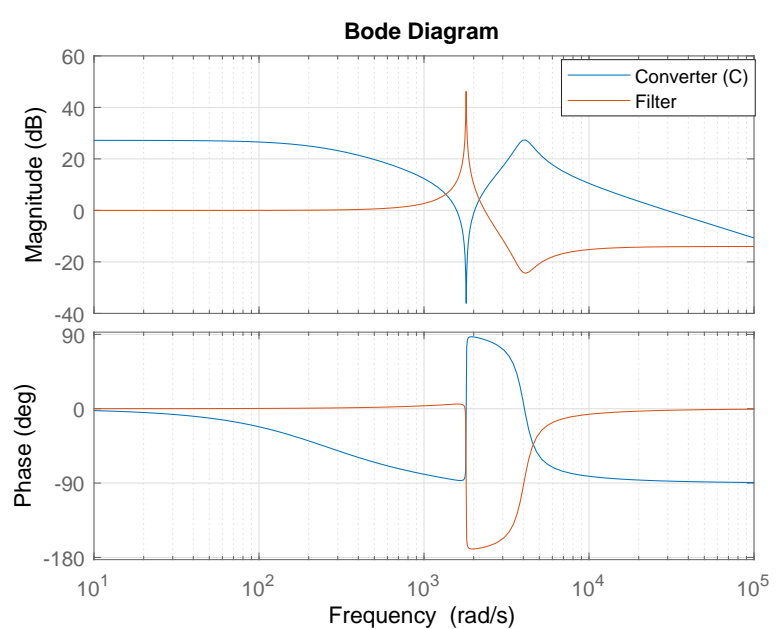

Fig. 5. Bode plots for the converter (blue) and the filter (orange).

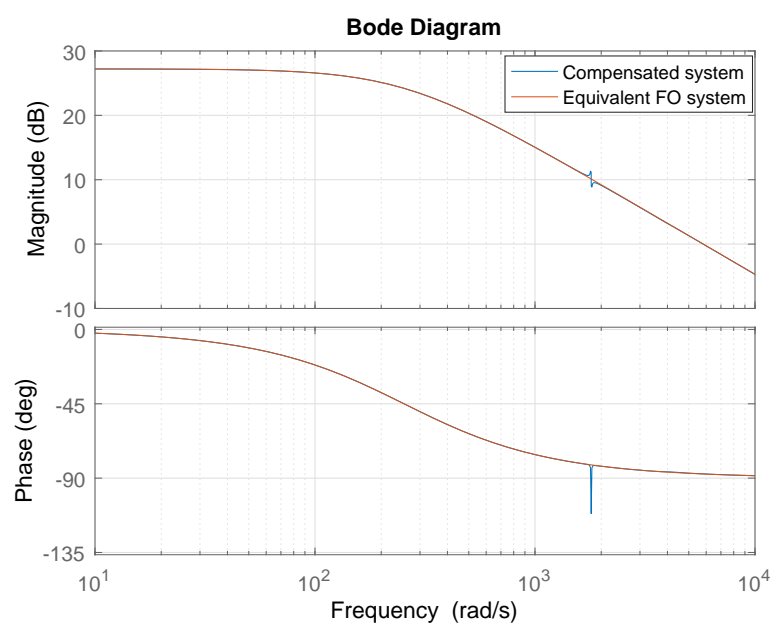

Fig. 6. Bode plot for the converter and filter system in open loop (blue) and equivalent simplified model $P_{r e d}$ (orange).

The Bode plot of the simplified first order model is also provided in Fig.6.

\section{B. Design of PI+CI with variable reset ratio}

The compensated first order system is used for the design of the PI+CI controller with variable reset ratio to obtain a flat response to step input as outlined in [11]. The design equations of the variable reset ratio controller are obtained by treating the error equation of the system as sequence of LTI system responses active between the successive reset instants. The derivation of the equations are not presented here and can be found in detail in [11]. Based on this framework, for a first order system defined as

$$
P_{\text {red }}(s)=\frac{b_{0}}{s+a_{0}} .
$$

with zero crossing reset, the error equation $\left(E_{r k}(s)\right)$ for reset instants defined by $k=0,1,2 \ldots$ is given by

$$
E_{r k}(s)=\frac{a_{0} w_{1}-b_{0} k_{i}\left(1-\rho_{r}\left(t_{k}\right)\right) x_{i}\left(t_{k}\right)}{s^{2}+\left(a_{0}+b_{0} k_{p}\right) s+b_{0} k_{i}}
$$


where $k_{p}$ and $k_{i}$ are the parameters of $P I_{b a s e}$. Equation (16) is obtained by solving (6) which is nothing but the solution of the base system (without reset) defined in the intervals of reset $\left(t_{k}, t_{k+1}\right]$. The solution of the base system is defined with $\mathbf{x}\left(\mathbf{t}_{\mathbf{k}}^{+}\right)$(the state values just after instance of reset $\mathrm{k}$ ) as initial value.

It can be seen from (16) that the error dynamics can be forced to zero by choosing a proper value for $\rho_{r}$ at the resetting instants. Therefore, value of $\rho_{r}$ to achieve a flat response is given by

$$
\rho_{r}\left(t_{k}^{+}\right)= \begin{cases}0 & k=0 \\ 1-\frac{a_{0} w_{10}}{b_{0} k_{i} x_{i}\left(t_{k}\right)} & k>0\end{cases}
$$

where $x_{i}\left(t_{k}\right)$ is the integrator value at reset instant. It should be noted that $\rho_{r}\left(t_{0}\right)=\rho_{r}(0)=0$ assuming that $t_{0}=0$. The value of $\rho_{r}$ from (17) appears to be varying but in real implementation will be constant after the first zero crossing. This is due to the fact that since a flat response is expected, after the first zero crossing the system is forced into steady state resulting in $x_{i}\left(t_{k}\right)=x_{i}\left(t_{k+1}\right)$.

The PI+CI controller design will be done as follows. First the base PI $\left(P I_{\text {base }}\right)$ parameters $\left(k_{p}\right.$ and $\left.k_{i}\right)$ are defined to have a very fast response with significant overshoot. The value of $\rho_{r}$ will then be calculated using (17) to ensure the flat response. The controller will then be realised as in Fig. 1. In the case of the boost converter defined in this problem the $P I_{b a s e}$ parameters $k_{p}$ and $k_{i}$ values were calculated to be 0.0348 and 38.125 respectively. The system was designed to have a settling time of $0.015 \mathrm{~s}$ and peak overshoot of $20 \%$. The $\rho_{r}$ value for the system was then calculated using (17) and found to be 0.3910 .

The PI+CI describing function is

$$
P I+C I(j \omega)=k_{p} \frac{j\left(w \tau_{i}+\frac{4}{I I} \rho_{r}\right)+1}{j \omega \tau_{i}}
$$

where $\tau_{i}$ is time constant of $P I_{b a s e}$ The describing function shows that the PI+CI always have a phase lead over the PI controller up to a frequency of $1 / \tau_{i}$ as highlighted in [12]. Informally speaking, this ensures that if the base PI system is stable the PI+CI system will also remain stable.

\section{RESULTS AND DISCUSSION}

The simulations results for the PI+CI-filter-boost converter system is presented here. The results from Simulink have been generated using the average model of the converter. The Fig.7 compares unit step response of system without filter for PI and PI+CI controller. The PI+CI has a definite performance improvement over the PI controller with reduced overshoot and faster settling time but flat response as expected is not obtained.

The Fig. 8 shows the unit step response with the filter in the system. The effect of filter can observed in the flat response to unit step for the PI+CI system. This is a significant performance improvement compared to the linear PI controller. The reset instance can be seen in Fig.9 which shows the control action of the controllers. The sharp reduction in the

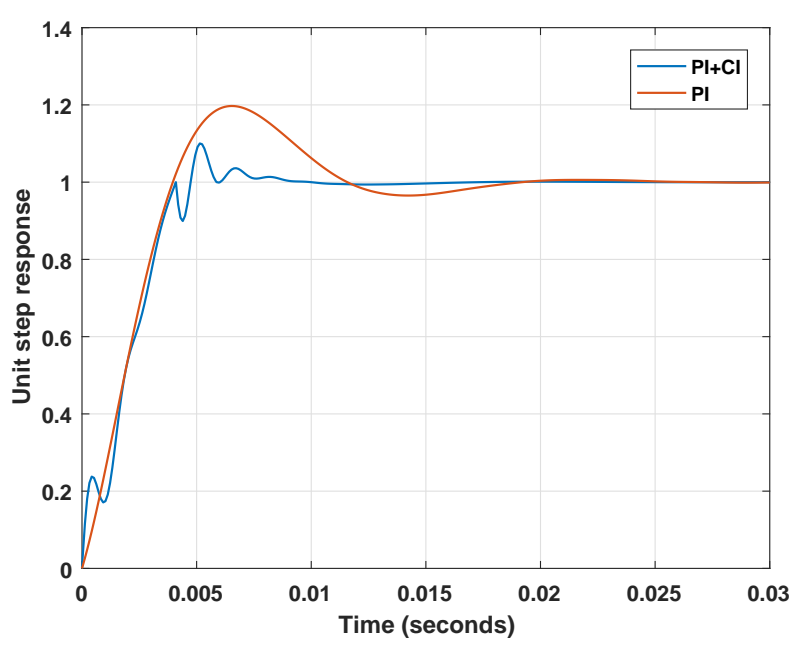

Fig. 7. Step response of the system with PI and PI+CI controller without filter.

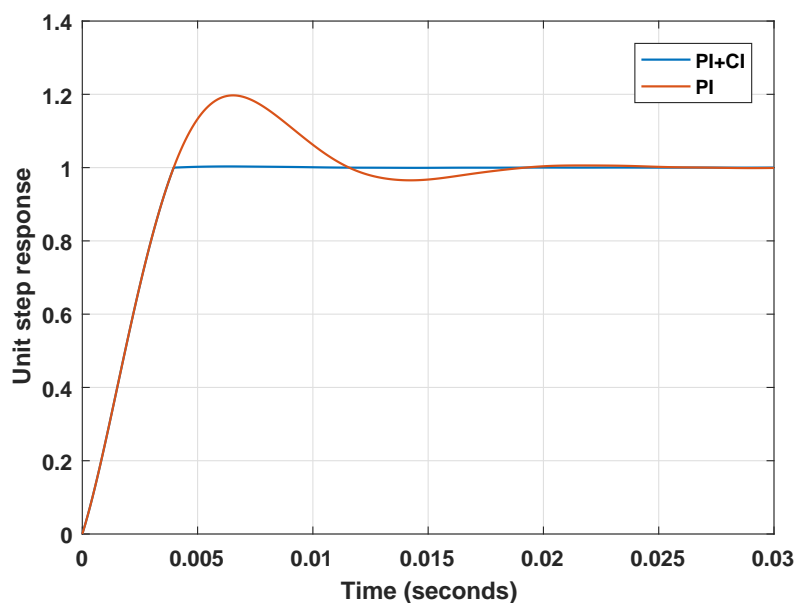

Fig. 8. Step response for the converter with PI+CI(blue) and PI (orange) controller.

controller output for PI+CI caused by the reset action drove the system to steady state after the first reset instant.

\section{CONClusion}

The PI+CI controller, based on simulation results, provides better performance over PI controller. The flat response observed in the results is impossible with a PI controller. Another advantage of the PI+CI controller is the relative simplicity in designing such a controller which is done using simple analytical equations. In the perspective of power converter this result is highly encouraging as it allows a fast response to input changes without introducing significant oscillation. This is very important especially when the converter is grid connected where such oscillation needs to be limited and fast response is desired. As mentioned before according to the authors knowledge such a resetting PI+CI controller has not been implemented for power converters. As such this offers lot of prospectives in terms of future work.

The areas that need investigation will be analysis of system 


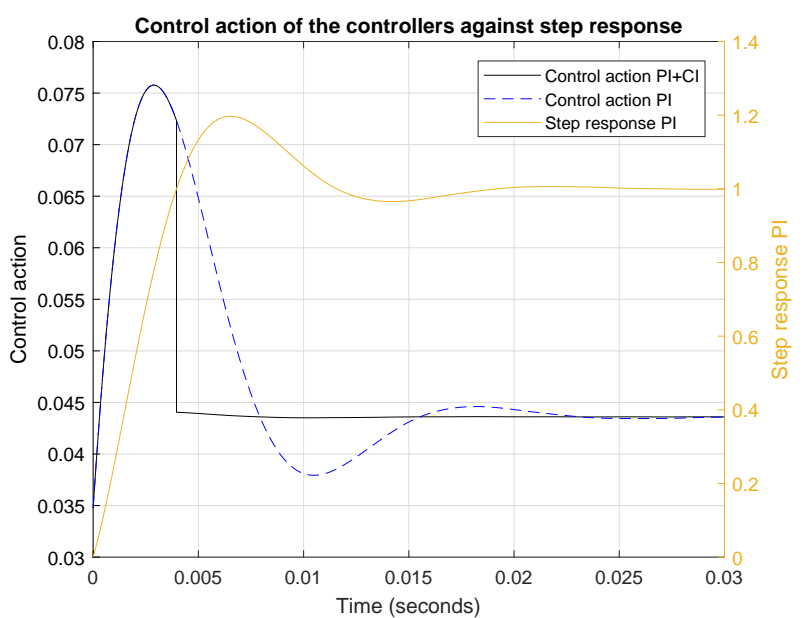

Fig. 9. Control action of PI+CI and PI controllers with the step response for the system with PI controller (orange) showing reset instant.

performance with the introduction of the switching ripples, experimental validation and paralleling of converters using this type of control. More stress need to be given in future work on the deeper analysis on the robustness and stability issues of such controller when applied to power converters.

\section{ACKNOWLEDGMENTS}

This work is done as part of project which has received funding from the European Union's Horizon 2020 research and innovation programme under the Marie Skłodowska Curie grant agreement No 675318 (INCITE).

\section{REFERENCES}

[1] P. Denholm, E. Ela, B. Kirby, and M. Milligan, "The Role of Energy Storage with Renewable Electricity Generation The Role of Energy Storage with Renewable Electricity Generation," Contract, vol. NREL/, no. January, pp. 1-53, 2010.

[2] H. Chen, T. N. Cong, W. Yang, C. Tan, Y. Li, and Y. Ding, "Progress in electrical energy storage system: A critical review," Progress in Natural Science, vol. 19, no. 3, pp. 291-312, 2009.

[3] L. Piris-Botalla, G. G. Oggier, A. M. Airabella, and G. O. Garca, "Extending the soft-switching operating range of a bi- directional three-port dc-dc converter," Revista Iberoamericana de Automtica e Informtica Industrial RIAI, vol. 13, no. 1, pp. 127 - 134, 2016.

[10] I. Horowitz and P. Rosenbaum, "Non-linear design for cost of feedback reduction in systems with large parameter uncertainty," International Journal of Control, no. 6, pp. 977-1001, jun.
[4] R. S. Ashok, Y. B. Shtessel, S. Member, and J. E. Smith, "Sliding Mode Control of Electric Power System Comprised of Fuel Cells , DC-DC Boost Converters and Ultracapacitors," no. 256, pp. 57865791, 2013

[5] R. S. Ashok, Y. B. Shtessel, and S. Member, "Sliding Mode Control of Electric Power System Comprised of Fuel Cell and Multiple-Modular DC-DC Boost Converters," no. 256, 2014.

[6] R. Venkataramanan, "Sliding mode control of power converters," Ph.D. dissertation, California Institute of Technology, 1986.

[7] A. Banos and A. Vidal, "Design of Reset Control Systems: The PI+CI Compensator," Journal of Dynamic Systems, Measurement, and Control, vol. 134, no. 5, p. 051003, 2012.

[8] J. C. Clegg, "A nonlinear integrator for servomechanisms," Transactions of the American Institute of Electrical Engineers, Part II: Applications and Industry, vol. 77, no. 1, pp. 41-42, 1958.

[9] K. R. Krishnan and I. M. Horowitz, "Synthesis of a non-linear feedback system with significant plant-ignorance for prescribed system tolerances," International Journal of Control, vol. 19, no. 4, pp. 689706, apr 1974.

[11] A. Baños and M. Davó, "Tuning of reset proportional integral compensators with a variable reset ratio and reset band," IET Control Theory and Applications, vol. 8, no. 17, pp. 1949-1962, 2014.

[12] A. Baños and A. Barreiro, Reset control systems. Springer Science \& Business Media, 2011

[13] G. Zhao and J. Wang, "On L2 gain performance improvement of linear systems with Lyapunov-based reset control," Nonlinear Analysis: Hybrid Systems, pp. 105-117, 2016.

[14] V. Ghaffari, P. Karimaghaee, and A. Khayatian, "Reset law design based on robust model predictive strategy for uncertain systems," Journal of Process Control, vol. 24, no. 1, pp. 261-268, 2014.

[15] S. van Loon, K. Gruntjens, M. Heertjes, N. van de Wouw, and W. Heemels, "Frequency-domain tools for stability analysis of reset control systems," Automatica, vol. 82, pp. 101-108, 2016.

[16] M. F. Heertjes, K. G. J. Gruntjens, S. J. L. M. van Loon, N. van de Wouw, and W. P. M. H. Heemels, "Experimental Evaluation of Reset Control for Improved Stage Performance," IFAC-PapersOnLine, no. 13, pp. 93-98, 2016.

[17] A. Vidal and A. Baños, "Reset compensation for temperature control: Experimental application on heat exchangers," Chemical Engineering Journal, vol. 159, no. 1, pp. 170-181, 2010.

[18] R. Goebel, R. G. Sanfelice, and A. R. Teel, Hybrid Dynamical Systems : Modeling, Stability, and Robustness. Princeton University Press.

[19] A. Baños, J. I. Mulero, A. Barreiro, and M. A. Davó, "An impulsive dynamical systems framework for reset control systems," International Journal of Control, vol. 89, no. 10, pp. 1985-2007, 2016.

[20] R. W. Erickson, Fundamentals of Power Electronics Fundamentals of Power Electronics. Kluwer Academic, 2002.

[21] S. Skogestad, "Simple analytic rules for model reduction and PID controller tuning," Modeling, Identification and Control, vol. 25, no. 2, pp. 85-120, 2004. 